\title{
The toric ideal of a matroid of rank 3 is generated by quadrics
}

\author{
Kenji Kashiwabara \\ Department of General Systems Studies, University of Tokyo, \\ 3-8-1 Komaba, Meguroku, Tokyo, 153-8902, Japan. \\ kashiwa@idea.c.u-tokyo.ac.jp
}

Submitted: Aug 27, 2008; Accepted: Feb 1, 2010; Published: Feb 15, 2010

Mathematics Subject Classification: 52B40

\begin{abstract}
White conjectured that the toric ideal associated with the basis of a matroid is generated by quadrics corresponding to symmetric exchanges. We present a combinatorial proof of White's conjecture for matroids of rank 3 by using a lemma proposed by Blasiak.
\end{abstract}

\section{Introduction}

The bases of a matroid have many good properties. Combinatorial optimization problems among them can be effectively solved. In this paper, we consider the conjecture about the bases of a matroid proposed by White [6]. While this conjecture has occasionally been stated in terms of algebraic expressions, it is closely related to combinatorial problems. Our proof adopts a combinatorial approach.

A matroid has several equivalent definitions. We define a matroid by a set of subsets that satisfies the exchange axiom. A family $\mathcal{B}$ of sets is the collection of bases of a matroid if it satisfies the exchange axiom given below.

(E) For any $X$ and $Y$ in $\mathcal{B}$, for every $a \in X$, there exists $b \in Y$ such that $X \cup\{b\}-\{a\}$ is in $\mathcal{B}$.

An element of $\mathcal{B}$ is called a base.

The exchange axiom is equivalent to the following stronger axiom, known as the symmetric exchange axiom.

(SE) For any $X$ and $Y$ in $\mathcal{B}$, for every $a \in X$, there exists $b \in Y$ such that $X \cup\{b\}-\{a\}$ and $Y \cup\{a\}-\{b\}$ are in $\mathcal{B}$.

The pair $X \cup\{b\}-\{a\}$ and $Y \cup\{a\}-\{b\}$ of bases is said to be obtained from the pair $X, Y$ of bases by a symmetric exchange. 
Let $M$ be a matroid on a ground set $E=\{1,2, \ldots, n\}$. For each base $B$ of $M$, we consider a variable $y_{B}$. Let $S_{M}$ be the polynomial ring $K\left[y_{B}: B\right.$ is a base of $\left.M\right]$ where $K$ is a field. Let $I_{M}$ be the kernel of the $K$-algebra homomorphism $\theta_{M}: S_{M} \rightarrow K\left[x_{1}, \ldots, x_{n}\right]$ such that $y_{B}$ is sent to $\Pi_{x \in B} x_{i} . I_{M}$ is a toric ideal (See [5]). White presented the following conjecture.

Conjecture 1. [6] For any matroid $M$ on $E$, the toric ideal $I_{M}$ is generated by the quadratic binomials $y_{X_{1}} y_{X_{2}}-y_{Y_{1}} y_{Y_{2}}$ such that the pair of bases $Y_{1}, Y_{2}$ can be obtained from the pair $X_{1}, X_{2}$ by a symmetric exchange.

Sturmfels [4] showed this conjecture for uniform matroids. Blasiak [1] showed this conjecture for graphic matroids.

In this paper, we prove White's conjecture for matroids of rank 3 or less.

\section{Preparation for proof}

Our main theorem is presented below.

Theorem 2. Conjecture 1 is true for matroids of rank 3 or less.

The problem, first described in algebraic terms, can be transformed into a combinatorial description.

We present the concept of a $k$-base graph. Let $M$ be a matroid on a ground set $E$ of size $n=r(M) k$, where $r(M)$ denotes the rank of $M$ and $k$ is a natural number. The $k$-base graph of $M$ has the set of all sets of $k$ disjoint bases as its vertex set. It has an edge connecting $\left\{X_{1}, \ldots, X_{k}\right\}$ and $\left\{Y_{1}, \ldots, Y_{k}\right\}$ if and only if $X_{i}=Y_{j}$ for some $i, j$. Such partitions are called adjacent.

Lemma 3. [1] Let $\mathcal{C}$ be a collection of matroids that is closed under deletions and additions of parallel elements. Suppose that for each $k \geqslant 3$ and for every matroid $M$ in $\mathcal{C}$ on a ground set of size $r(M) k$ the $k$-base graph of $M$ is connected. Then for every matroid $M$ in $\mathcal{C}, I_{M}$ is generated by quadratic binomials.

Since, for a matroid $M$ of rank at most 3, any quadratic binomial in $I_{M}$ is in the ideal of generated by quadratic binomials corresponding to symmetric exchanges, we have the following corollary.

Corollary 4. Let $\mathcal{C}$ be a collection of matroids of rank at most 3 that is closed under deletions and additions of parallel elements. Suppose that for each $k \geqslant 3$ and for every matroid $M$ in $\mathcal{C}$ on a ground set of size $r(M) k$ the $k$-base graph of $M$ is connected. Then for every matroid $M$ in $\mathcal{C}, I_{M}$ is generated by quadratic binomials corresponding to symmetric exchanges.

The deletion of a matroid of rank 3 is of rank at most 3. Adding parallel elements to a matroid of rank 3 does not change its rank. Therefore, the class of matroids of rank 3 or less satisfies the assumption for the class of matroids in the lemma. To prove the 
conjecture for matroids of rank 3 or less, it suffices to prove the connectivity of the $k$-base graph in the lemma.

The conjecture for matroids of rank 2 is relatively easy to show. In fact, Blum [2] proposed a base-sortable matroid and showed that the toric ideal of a base-sortable matroid has a square-free quadratic Gröbner basis. This fact implies that Conjecture 1 is true for the matroids of rank 2.

To prove Theorem 2, it suffices to prove the next theorem by Corollary 4 .

Theorem 5. The k-base graph of a matroid of rank 3 is connected.

The remainder of this paper is devoted to the proof of this theorem.

We consider a matroid $M$ of rank 3 on a ground set $E$ of size $n=3 k$. Note that, on a ground set of size at least 9 , when one partition is obtained by a symmetric exchange from the other because these two partitions have a common base, the two partitions are adjacent in the $k$-base graph.

We show that, for any two vertices of the $k$-base graph, one vertex connects to the other. Each vertex in the $k$-base graph corresponds to a partition consisting of bases. We call one partition of bases the initial blue partition and the other the initial red partition. In the figures shown below, a base in the initial blue partition and red partition is colored blue and red, respectively. Moreover, a partition that is known to be connected to the initial blue (red) in the $k$-base graph is colored a shade of blue (red), and we also call it a blue (red) partition. Moreover we call a base in a blue (red) partition a blue (red) base. A base that is not known to be contained in any blue or red partition is colored green in the figures in the sequel.

We show that the initial blue and red partitions are connected in the $k$-base graph. That is, we show that there exists a base that is red and blue. We prove the connectivity of the $k$-base graph of a matroid of rank 3 by a mathematical induction on the size of the ground set. We describe the base case of the induction in Section 3 and describe the step in Section 4.

\section{Base case of induction}

In this section, we show the base case of the induction in the proof of Theorem 5 . We show the connectivity of the 3-base graph of a matroid of rank 3 and size 9 . In this section, we consider a matroid such that its bases are of size 3 and its ground set is of size 9 .

Consider the initial blue and red partitions, consisting of bases of size 3 , on a set of size 9. Assume that there exists no base that belongs to both partitions. Then, the possible positions between the initial blue and red partitions are essentially classified into three patterns as shown in Figure 1 up to permutation of the vertices. We consider each case in the following three subsections.

Note that the matroid may have more bases than the bases that belong to the initial red or blue partitions. In other words, the bases appearing in the figure are not all of the bases of the matroid. 

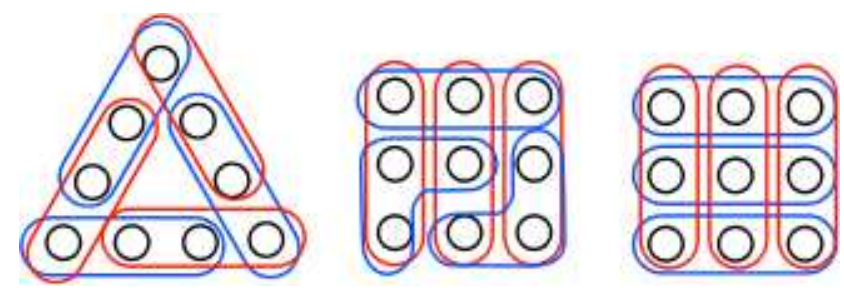

Figure 1: Three patterns on the set of size 9

\subsection{First case of size 9}

We consider the first case of of a matroid on a set of size 9. We use letters to label the vertices, as in the first figure of Figure 2.
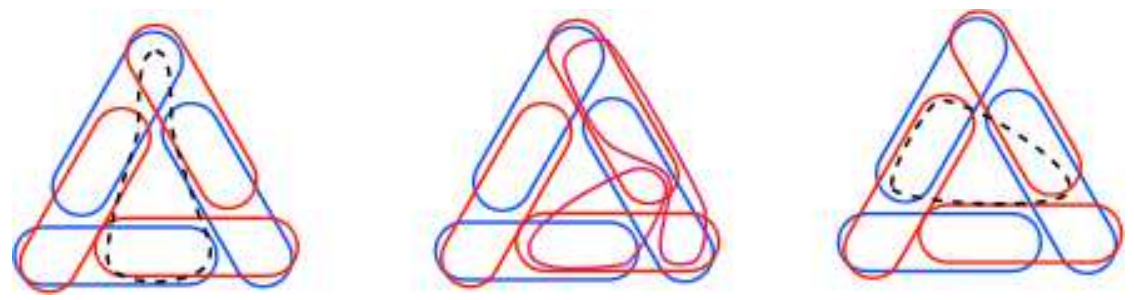

Figure 2: First case of size 9

If aef is a base, $\{\mathbf{b c d}$, aef, $\mathbf{i h g}\}$ is a partition of bases, that contains both a blue base and a red base. Therefore this partition is adjacent to both the initial blue and red partitions in the 3-base graph. Therefore, we know that aef cannot be a base. In the first figure of Figure 2, the black dotted line implies that aef is not a base. Similarly, bcg cannot be a base.

For red bases aih and $\mathbf{e f g} \mathbf{- g}$, by the exchange axiom, there exist red bases (aef and ihg), (efi and $\mathbf{a f g}$ ), or (efh and aig). Because aef is not a base, either (efi and $\mathbf{a f g}$ ) or (efh and aig) are bases. Without loss of generality, we may assume that efh and aig are red bases (the second figure of Figure 2). Recall that such a base is colored by a color similar to red in the figures.

If $\mathbf{b c h}$ is a base, $\{\mathbf{a i g}, \mathbf{b c h}, \mathbf{d e f}\}$ is a partition, that contains both a blue base and a red base. Therefore we may assume that bch is not a base (the third figure of Figure 2).

Moreover, by the exchange axiom for blue bases abc - a and ghi, there exists a blue base (bcg and aih), (bch and aig), or (bci and ahg). Because we already know that bcg and bch are not bases, bci and ahg are bases (the second figure of Figure 3).

Next, we consider the exchange axiom for blue bases ghi and def - $\mathbf{d}$. Then, there exists a blue partition containing $\mathbf{e f g}$, efh, or efi. When either efh or efg is a base, it also belongs to a red partition. When efi is a base, $\{\mathbf{a h g}, \mathbf{b c d}$, ief $\}$ is a partition, that contains 

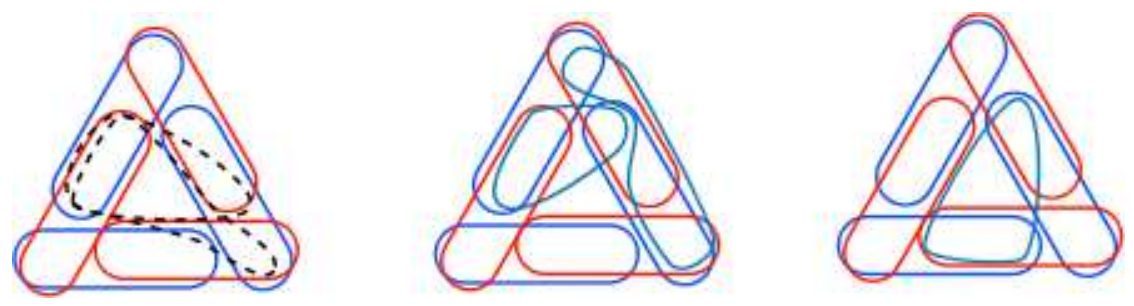

Figure 3: First case of size 9: continued

both a blue base and a red base (the third figure of Figure 3). We have completed the proof of the first case of size 9 .

\subsection{Second case of size 9}

We consider the second case of a matroid on a set of size 9 .
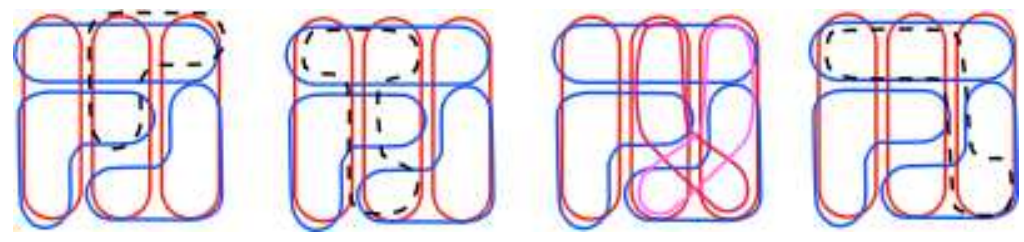

Figure 4: Second case of size 9

When bce is a base, $\{\mathbf{a d g}, \mathbf{b c e}, \mathbf{f h i}\}$ is a partition, which contains both a blue base and a red base. Therefore we may assume that bce is not a base (the first figure of Figure 4).

Similarly, if abh is a base, $\{\mathbf{d e g}, \mathbf{a b h}, \mathbf{c f i}\}$ is a partition, that contains both a blue base and a red base. Therefore, abh is not a base (the second figure of Figure 4).

By the exchange axiom for red bases cfi and beh - $\mathbf{h}$, there exists a red base (bec and fhi), (bef and chi), or (bei and hcf). Since bce is not a base, either bef or bei is a base. Without loss of generality, we may assume that bei and hcf are red bases (the third figure of Figure 4).

When abi is a base, $\{\mathbf{d e g}, \mathbf{a b i}, \mathbf{c f h}\}$ is a partition, that contains both a blue base and a red base. Therefore we may assume that abi is not a base (the fourth figure of Figure 4).

By the exchange axiom for blue bases fhi and $\mathbf{a b c}-\mathbf{c}$, there exists a blue base abf, $\mathbf{a b h}$, or abi. Since abh and abi are not bases, abf is a blue base (the first figure of Figure 5).

By the exchange axiom for red base beh and blue base abc - a, either bce or bch is a base. Since bce is not a base, bch must be a base (the second figure of Figure 5). 

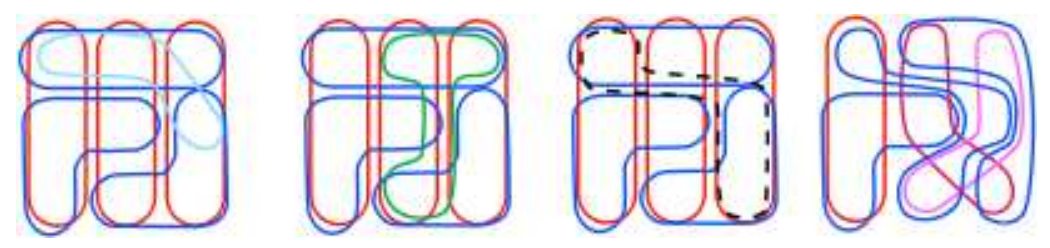

Figure 5: Second case of size 9: continued

This base is not known to belong to some red or blue partition, and therefore it is colored green in the figure.

When afi is a base, $\{\mathbf{b c h}, \mathbf{a f i}, \mathbf{d e g}\}$ is a blue partition. Therefore the relationship between this blue partition and the initial red partition $\{\mathbf{a d g}, \mathbf{b e h}, \mathbf{c f i}\}$ is isomorphic to the first case of size 9. Therefore this case results in the first case. Therefore we may assume that afi is not a base (the third figure of Figure 5).

In other words, we know that abi and afi are not bases. Therefore we have $r(\mathbf{a b i})=$ $r(\mathbf{a f i})=2$. Since $\mathbf{a b f}$ is a base, we have $r(\mathbf{a b f i})=3$. Then, by submodularity, $1 \leqslant r(\mathbf{a i}) \leqslant$ $r(\mathbf{a b i})+r(\mathbf{a f i})-r(\mathbf{a b f i})=2+2-3=1$. Therefore we have $r(\mathbf{a i})=1$. Therefore $\mathbf{a}$ and i are parallel.

Since $\mathbf{a}$ and $\mathbf{i}$ are parallel, $\{\mathbf{a f h}, \mathbf{b c i}, \mathbf{d e g}\}$ is a blue partition. The position between this partition $\{\mathbf{a f h}, \mathbf{b c i}, \mathbf{d e g}\}$ and red partition $\{\mathbf{a d g}, \mathbf{b e i}, \mathbf{c f h}\}$ appears in the first case of size 9(the fourth figure of Figure 5).

\subsection{Last case of size 9}

We consider the last case of a matroid on a set of size 9 , illustrated in the third figure of Figure 1.
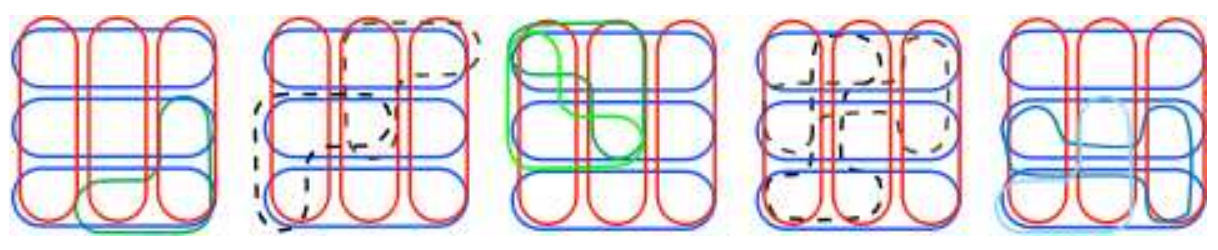

Figure 6: Last case of size 9

By the exchange axiom for cfi and ghi - g, either chi or fhi is a base. Without loss of generality, we may assume that fhi is a base (the first figure of Figure 6).

When bce is a base, $\{\mathbf{a d g}, \mathbf{b c e}, \mathbf{f h i}\}$ is a red partition. This case results in the second case of a matroid on a set of size 9 . Therefore we may assume that bce is not a base. Similarly, we may assume that deg is not a base (the second figure of Figure 6). 
By the exchange axiom for $\mathbf{a b c}$ and $\mathbf{b e h} \mathbf{-} \mathbf{h}$, either abe or bce is a base. Since bce is not a base, abe is a base. Similarly, ade is a base (the third figure of Figure 6).

By resulting in the second case, cdf and bgh are not bases (the fourth figure of Figure $6)$.

Then, similarly, by the exchange axiom for beh and ghi - i, egh is a base. Moreover, by the exchange axiom for $\mathbf{c f i}$ and def - e, dfi is a base. Then $\{\mathbf{a b c}, \mathbf{e g h}, \mathbf{d f i}\}$ is a blue partition. This case results in the second case (the fifth figure of Figure 6).

We have completed the proof of the last case when the ground set is of size 9 .

\section{Inductive step}

In the previous section, we confirmed that the $k$-base graph of a matroid of rank 3 and size 9 is connected. In this section, we show the inductive step: we will prove Theorem 5 on a ground set of size $n=3 k, k>3$, assuming the theorem for matroids on sets of size less than $n$. Let $\left\{B_{1}, \ldots, B_{k}\right\}$ be the initial blue partition.

We separate the inductive step into two cases according to the relative position between the initial blue partition and the initial red partition. In one case, for some two bases in the initial blue partition, some red base is included in the union of the two blue bases. In the other case, there exists no red base included in the union of any two blue bases.

We use the following well-known lemma, which is a consequence of the matroid partition theorem [3].

Lemma 6. Consider a matroid of rank $r$ on $E$ where $k$ is an integer and $|E|=k r$. There exists a partition consisting of bases if and only if $k r(X) \geqslant|X|$ for any $X \subseteq E$.

\subsection{First case in inductive step}

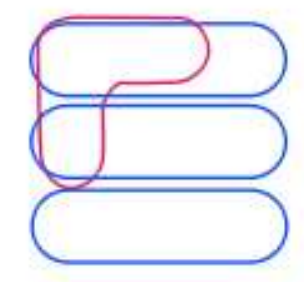

Figure 7: First case in inductive step

Denote $B_{1}=$ abc and $B_{2}=$ def. Let abd be a base in the initial red partition (Figure 7).

Let $B_{i}=$ ghi be a base in the initial blue partition with $i \geqslant 3$. 
Lemma 7. Consider the matroid obtained by restricting the given matroid of rank 3 to cefghi.

There exists a partition consisting of two bases included in cefghi if and only if it satisfies the following two conditions.

Condition 1: $r(\mathbf{c e g}), r(\mathbf{c e h}), r(\mathbf{c e i}), r(\mathbf{c f g}), r(\mathbf{c f h}), r(\mathbf{c f i}) \geqslant 2$.

Condition 2: $r($ cefgh $)=r($ cefgi $)=r($ cefhi $)=3$.

Proof. Assume that there exists a partition of two bases on cefghi. Then $2 r(X) \geqslant|X|$ for any $X \subseteq$ cefghi by Lemma 6 .

When $|X|=3, r(X) \geqslant 2$ because $r$ is integer-valued. Therefore Condition 1 holds.

When $|X|=5, r(X) \geqslant 3$ because $r$ is integer-valued. Therefore Condition 2 holds.
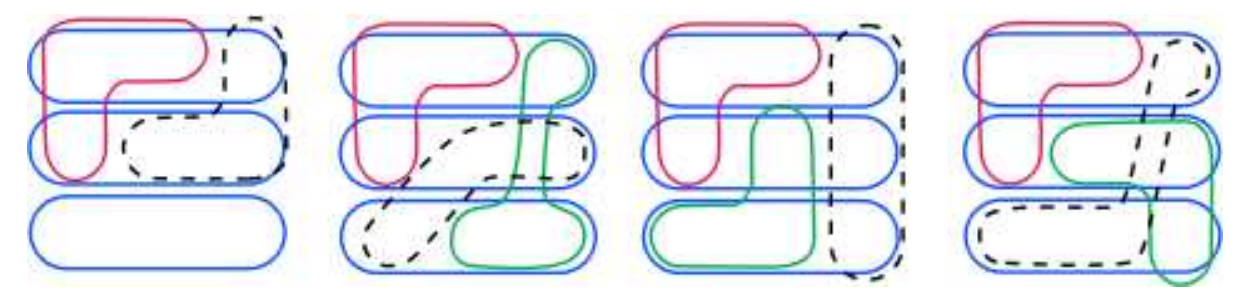

Figure 8: Bases and non-bases in the proof

Conversely, we assume Condition 1 and Condition 2. Then $2 r(X) \geqslant|X|$ for any $X \subseteq$ cefghi. Note that all other 2 and 3 element subsets not mentioned in Conditions 1 and 2 automatically have a sufficiently large rank because ghi and ef are independent. Therefore there exists a partition consisting of two bases included in cefghi by Lemma 6.
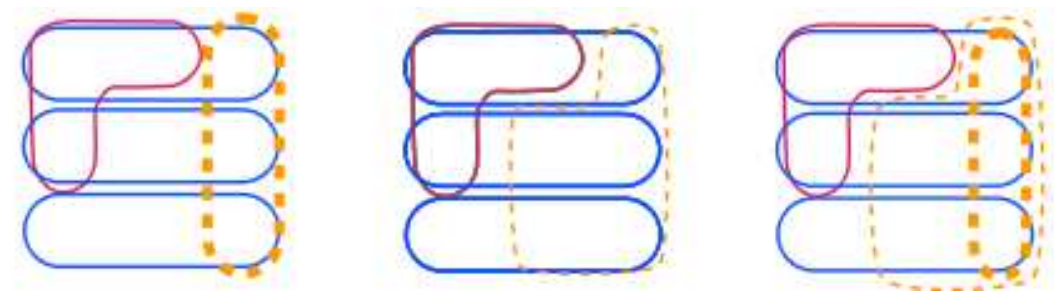

Figure 9: Three cases of flats

If there exists a partition consisting of two bases included in cefghi, it results in the case of the ground set of size 9. Therefore we may assume that there exists no partition consisting of two bases included in cefghi. By Lemma 7, at least one among Condition 1 and Condition 2 is not satisfied. 
If Condition 1 does not hold, we may assume that cfi is of rank 1 without loss of generality (the first figure or the third figure in Figure 9). Then, any set that includes cfi as a proper subset on cefghi is of rank more than 1 because it contains an independent set of size 2. In the figures, the set enclosed by a dashed orange line represents a flat on the matroid on cefghi. The set enclosed by a thick line implies a flat of rank 1 . The set enclosed by a thin line implies a flat of rank 2. If Condition 2 does not hold, there exists a set of rank 2 and size 5 (the second figure or the third figure in Figure 9). Then, any set that includes this set as a proper subset on cefghi is of rank 3 because it would have contained a base, that is, this set is a flat of rank 2 on the matroid restricted to cefghi.

By changing $B_{i}$, we investigate the matroid on $E$ as follows. We suppose that the matroid on $B_{1} \cup B_{2} \cup B_{i}$ for all $i \geqslant 3$ does not satisfy Condition 1 . There exists $A \subseteq E$-abd of rank 1 and size $k=n / 3$ since parallel relation induces an equivalence class. On the other hand, by Lemma 6 to the initial red partition, we have $(k-1) r(A) \geqslant|A|$ for any $A \subseteq E-$ abd. Therefore every $A \subseteq E-\operatorname{abd}$ of size $k=n / 3$ is of rank at least 2 , a contradiction.

We suppose that the matroid on $B_{1} \cup B_{2} \cup B_{i}$ for all $i \geqslant 3$ does not satisfy Condition 2. There exists $A \subseteq E-$ abd of rank 2 and size $2 k-1$ by submodularity and $r(\mathbf{c e f})=2$. On the other hand, by Lemma 6 to the initial red partition, we have $(k-1) r(A) \geqslant|A|$ for any $A \subseteq E-$ abd. Therefore every $A \subseteq E-$ abd of size $k=2 k-1$ is of rank 3 , a contradiction.
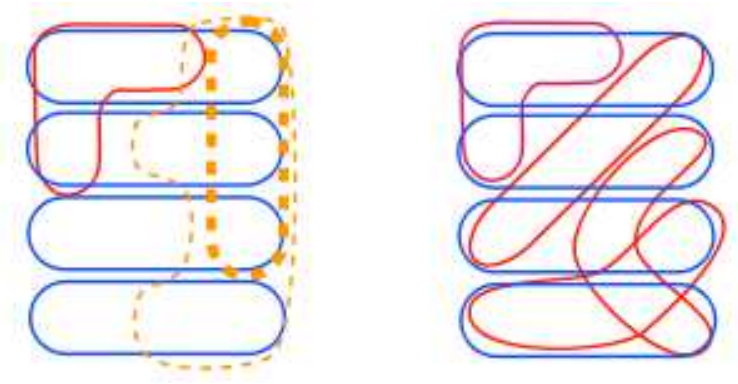

Figure 10: Flats and a partition on 12-set

Therefore we may assume that the matroid on $B_{1} \cup B_{2} \cup B_{i}$ for some $i \geqslant 3$ satisfies Condition 1 and does not satisfy Condition 2, and the matroid on $B_{1} \cup B_{2} \cup B_{j}$ for some $j \geqslant 3$ satisfies Condition 2 and does not satisfy Condition 1 . Denote $B_{i}=$ ghi and $B_{j}=\mathbf{j k k}$. We consider the matroid restricted to cefghijkl. We may assume that $\mathbf{c f i}$ is a flat of rank 1 and cefikl is a flat of rank 2 on cefghijkl (the left-hand side of Figure 10). Note that the flat of rank 1 is included in the flat of rank 2 by submodularity.

Then a set, of size 3, consisting of one of cfi, one of ekl, and one of $\mathbf{g h j}$ must be a base because of the following reason. If this set is dependent, it contradicts the fact that cfi and cefikl are flats by submodularity. Therefore it is independent. Therefore ceg, fhl, and $\mathbf{i j k}$ are bases. We have a red partition consisting of these bases and $B_{k}$ for $i \neq 1,2, i, j$ 
(the right-hand side of Figure 10). Similarly, any set of size 3 consisting of one of cfi, one of ekl, and one of ghj is red.

It remains to show that this partition and the initial blue partition are connected.

By the exchange axiom for blue bases $\mathbf{j k l}$ and ghi - i, there exist blue bases (ghj and $\mathbf{i k l})$, (ghk and $\mathbf{i j l}$ ), or (ghl and $\mathbf{i j k})$. When ghj and $\mathbf{i k l}$ are bases,it contradicts $r(\mathbf{i k l})=2$. When $\mathbf{g h l}$ is a base, $\{\mathbf{a b c}, \mathbf{d e f}, \mathbf{g h l}, \mathbf{i j k}\}$ is a partition using red base $\mathbf{i j k}$ and blue base ghl. When ghk is a base, $\{\mathbf{a b c}, \mathbf{d e f}, \mathbf{g h k}, \mathbf{i j l}\}$ is a partition using red base $\mathbf{i j l}$ and blue base ghk.

\subsection{Second case in inductive step}

We consider the second case in the inductive step, that is, any base in the initial red partition cannot be included in any two bases in the initial blue partition. We only have to consider the case with three bases $B_{1}=\mathbf{a b c}, B_{2}=$ def, $B_{3}=$ ghi in the initial blue partition and a base adg in the initial red partition as shown in Figure 11. We can assume that there exists no partition on bcefhi by the base case of the induction.

By the exchange axiom between abc and $\mathbf{a d g}-\mathbf{g}$, we may assume that there exists a green base abd without loss of generality. When there exists a partition containing abd, it connects both the initial blue and red partitions on the $k$-base graph by the first case in the inductive step. Therefore we can assume that there exists no partition on $E-\mathbf{a b d}$.

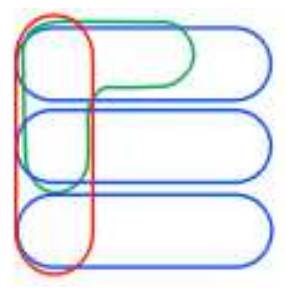

Figure 11: Second case in inductive step

Then, by Lemma 6 , there exists $A \subseteq E-$ abd of (Case I) rank 1 and size $k=n / 3$, or (Case II) rank 2 and size $2 k-1=2 n / 3-1$.

Case I: case where there exists a set $A$ of rank 1 and size $k$ in $E-\mathbf{a b d}$.

Then $A$ contains c because $A$ should intersect abc in the initial blue partition. Similarly, $A$ contains $\mathbf{g}$ because $A$ should intersect adg in the initial red partition. Since the rank of $\mathbf{c g}$ is $1, \mathbf{c}$ and $\mathbf{g}$ are parallel. Since $\mathbf{a b g}$ and $\mathbf{c h i}$ are exchangeable with abc and ghi, abg is a blue base, which results in the first case of the induction.

Case II: case where there exists a set $A \subseteq E-\operatorname{abd}$ of rank 2 and size $2 k-1$. Moreover we assume that the condition of Case I is not satisfied. Note that $A \cap B_{1}=\mathbf{c}$ and $\left|A \cap B_{k}\right|=$ 2 for any $k \geqslant 2$. Since $A$ intersects any base in the initial red partition, $|A \cap \mathbf{a d g}| \geqslant 1$. Since $A \cap \mathbf{a d}=\emptyset$, we have $\mathbf{g} \in A$. Since $\left|A \cap B_{3}\right|=2, A \cap\left(B_{1} \cup B_{2} \cup B_{3}\right)=\mathbf{c e f g h}$ or cefgi. We assume $A \cap\left(B_{1} \cup B_{2} \cup B_{3}\right)=$ cefgh without loss of generality. We have 
$r(A \cup \mathbf{b}-\mathbf{g})=3$ because $r(A \cup \mathbf{b}-\mathbf{g})=2$ contradicts the existence of the initial red partition and $|A \cup \mathbf{b}-\mathbf{g}|=2 k-1$ by Lemma 6 . Since there exists no partition on bcefhi, there exists a set $C \subseteq$ bcefhi of (Case II-1) rank 1 and size 3, or (Case II-2) rank 2 and size 5 by Lemma 6 .

Case II-1: case where $C \subseteq$ bcefhi is of rank 1 and size 3 .

When $\mathbf{b} \in C, \mathbf{b}$ is parallel to $\mathbf{e} \in A$ or $\mathbf{f} \in A$, which contradicts $r(A \cup \mathbf{b}-\mathbf{g})=3$. Therefore we have $\mathbf{c}=B_{1} \cap C$. On the other hand, we have $C \cap B_{3}=\mathbf{h}$ because $C \cap B_{3} \subseteq \mathbf{h i}$ and $C \cap B_{3} \subseteq A \cap B_{3}=\mathbf{g h}$. Therefore $C=\mathbf{c e h}$ or $C=\mathbf{c f h}$. We can assume that $\mathbf{c f h}$ is of rank 1 without loss of generality. Since the condition of Case I is not satisfied, there exists $i \geqslant 4$ such that $B_{i}$ has no element parallel to $\mathbf{c}$. Denote $B_{i}=\mathbf{j k l}$. Then $\mathbf{c f h}$ is a flat of rank 1 on the matroid on bcefhijkl. Let cefhkl be a flat of rank 2 on the matroid on bcefhijkl without loss of generality because of $\mathbf{g} \in A$ (Figure 12). Note that cefhkl does not span $\mathbf{b}$ because of $r(A \cup \mathbf{b}-\mathbf{g})=3$.

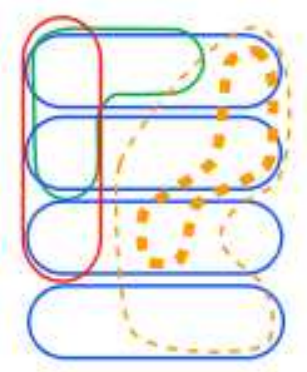

Figure 12: Flats on bcefhijkl

Then bce, fil, and hjk are bases. Therefore $\{\mathbf{a d g}, \mathbf{b c e}, \mathbf{f i l}, \mathbf{h} \mathbf{j k}\}$ is a partition on the 12 -set. Since the position between this partition and $\left\{B_{1}, B_{2}, B_{3}, B_{i}\right\}$ results in the first case in inductive step, abd is a blue base.

Case II-2: case where $C \subseteq$ bcefhi is of rank 2 and size 5 .

By the submodularity $r($ cefh $)+r($ cefghi $) \leqslant r($ cefhi $)+r($ cefgh $)$, we have $r($ cefhi $)=3$. Therefore $C \neq$ cefhi. Similarly, we have $C \neq$ bcefi. When $C=$ bcefh, we have $r(\mathbf{b c e f g h})=2$ by the submodularity $r(\mathbf{b c e f g h})+r(\mathbf{c e f h}) \leqslant r(\mathbf{c e f g h})+r(\mathbf{b c e f h})$. Then, by Lemma 6 , the existence of the initial red partition contradicts $r(A \cup \mathbf{b}-\mathbf{g})=2$ and $|A \cup \mathbf{b}-\mathbf{g}|=2 k-1$. Therefore we can assume $C=\mathbf{b c f h i}$ without loss of generality. Since $r(\mathbf{b c f h i})=2, r(\mathbf{c e f g h})=2$, and $r(\mathbf{b c e f g h i})=3$, we have $r(\mathbf{c f h})=1$ by submodularity, which results in Case II-1.

We have completed the proof of Theorem 5. 


\section{References}

[1] Jonah Blasiak, The Toric Ideal of a Graphic Matroid is generated by Quadrics, Combinatorica, Vol. 28(3), 283-297, 2008.

[2] Stefan Blum, Base-sortable Matroids and Koszulness of Semigroup Rings, European J. Combin., Vol. 22, 937-951, 2001.

[3] Jack Edmonds, Minimum partition of a matroid into independent subsets, Journal of Research of the National Bureau of Standards 69B, 67-72, 1965.

[4] Bernd Sturmfels, Gröbner Bases and Convex Polytopes, American Mathematical Society, University Lecture Series, Vol. 8, Providence, RI, 1995.

[5] Bernd Sturmfels, Equations Defining Toric Varieties. Proc. Symposia in Pure, American Mathematical Society, 437-449, 1997.

[6] Neil White, A Unique Exchange Property for Bases. Linear Algebra Appl., Vol. 31, 81-91, 1980. 